\title{
C. Elegans Fatty Acid Two-Hydroxylase Regulates Intestinal Homeostasis by Affecting Heptadecenoic Acid Production
}

\author{
Yuanbao Lia Chunxia Wang ${ }^{\mathrm{a}}$ Yikai Huang ${ }^{\mathrm{b}}$ Rong Fu ${ }^{\mathrm{a}}$ Hanxi Zheng ${ }^{\mathrm{b}}$ Yi Zhu $^{\mathrm{a}}$ \\ Xiaoruo Shi ${ }^{b}$ Prashanth K Padakantic Zhude Tu ${ }^{c}$ Xiong Sub,d Huimin Zhanga \\ ajiangsu Key Laboratory of Infection and Immunity, Institutes of Biology and Medical Sciences, \\ Soochow University, Suzhou, 'bepartment of Biochemistry and Molecular Biology, Soochow University \\ Medical College, Suzhou, China, 'Department of Radiology, Washington University School of Medicine, \\ St Louis, MO, dCenter for Human Nutrition, Washington University School of Medicine, St. Louis, MO, \\ USA
}

\section{Key Words}

C. elegans $\bullet$ Fatty acids $\bullet \mathrm{FA} 2 \mathrm{H} \cdot 2$-hydroxylation $\bullet$ Heptadecenoic acid

\begin{abstract}
Background/Aims: The hydroxylation of fatty acids at the C-2 position is the first step of fatty acid $\alpha$-oxidation and generates sphingolipids containing 2-hydroxy fatty acyl moieties. Fatty acid 2-hydroxylation is catalyzed by Fatty acid 2-hydroxylase (FA2H) enzyme. However, the precise roles of $\mathrm{FA} 2 \mathrm{H}$ and fatty acid 2-hydroxylation in whole cell homeostasis still remain unclear. Methods: Here we utilize Caenorhabditis elegans as the model and systemically investigate the physiological functions of FATH-1/C25A1.5, the highly conserved worm homolog for mammalian FA2H enzyme. Immunostaining, dye-staining and translational fusion reporters were used to visualize FATH-1 protein and a variety of subcellular structures. The "click chemistry" method was employed to label 2-OH fatty acid in vivo. Global and tissue-specific RNAi knockdown experiments were performed to inactivate FATH-1 function. Lipid analysis of the fath- 1 deficient mutants was achieved by mass spectrometry. Results: $C$. elegans FATH-1 is expressed at most developmental stages and in most tissues. Loss of fath-1 expression results in severe growth retardation and shortened lifespan. FATH-1 function is crucially required in the intestine but not the epidermis with stereospecificity. The "click chemistry" labeling technique showed that the FATH-1 metabolites are mainly enriched in membrane structures preferable to the apical side of the intestinal cells. At the subcellular level, we found that loss of fath-1 expression inhibits lipid droplets formation, as well as selectively disrupts peroxisomes and apical endosomes. Lipid analysis of the fath-1 deficient animals revealed a significant reduction in the content of heptadecenoic acid, while other major FAs remain unaffected. Feeding of exogenous heptadecenoic acid (C17:1), but not oleic acid (C18:1), rescues the global and subcellular defects of fath-1 knockdown worms. Conclusion: Our study revealed

Xiong Su

and Huimin Zhang

Department of Biochemistry and Molecular Biology, Soochow University Medical College

Soochow University, No. 199 Ren-Ai Road, Suzhou, Jiangsu, 215123 (China)

Tel. +86-512-65882162, E-Mail xsu@suda.edu.cn; zhanghuimin@suda.edu.cn
\end{abstract}


that FATH-1 and its catalytic products are highly specific in the context of chirality, C-chain length, spatial distribution, as well as the types of cellular organelles they affect. Such an unexpected degree of specificity for the synthesis and functions of hydroxylated FAs helps to regulate protein transport and fat metabolism, therefore maintaining the cellular homeostasis of the intestinal cells. These findings may help our understanding of $\mathrm{FA} 2 \mathrm{H}$ functions across species, and offer potential therapeutical targets for treating $\mathrm{FA} 2 \mathrm{H}$-related diseases.

(C) 2018 The Author(s)

Published by S. Karger AG, Basel

\section{Introduction}

Fatty acids (FAs) are one of the most common components of biological lipid species. FAs with diverse chemical structures are physiologically important in a variety of cellular functions including energy storage, membrane structure, trafficking and signaling [1]. Lipidomic analysis revealed the presence of many substituted FAs, such as hydroxylated FAs (OH-FAs) in animal lipids, whose cellular functions have not been well studied. Fatty acid 2-hydroxylase (FA2H) introduces a hydroxyl group specifically at the second carbon of FA substrates. Lipid species containing 2-OH FAs have unique functions in membrane homeostasis and cell signaling that could not be substituted by non-hydroxy counterparts $[2,3]$. FA2 $\mathrm{H}$ is essential for the normal functioning and differentiation of many cell types in mammals [4]. Mutations in the gene encoding FA2H are often associated with inherited human disorders such as HSP (hereditary spastic paraplegia) [5]. However, a complete picture of the cellular mechanisms of the FA2H functions is still not clear.

Fatty acid two-hydroxylase 1 (FATH-1) is the sole C. elegans homolog for mammalian $\mathrm{FA} 2 \mathrm{H}$, whose corresponding gene number is C25A1.5 [6, 7]. There have been a few isolated reports mentioning the function of FATH-1 in C. elegans. For example, fath-1/c25a1.5 inactivation by RNAi causes larval arrest and enhances dauer formation [6, 8]. FATH-1 was also found in a DAF-7/TGF- $\beta$ interactive network. It was proposed to physically interact with DAF-3/SMAD4 and repress dauer formation [8]. In addition, fath-1 was identified in a screen for genes affecting tubular polarity of the intestine [9]. An independent study identified fath1 among several other genes involved in fatty acid biosynthesis as a regulator of p38 MAPK pathway and intestinal innate immunity [10]. Finally, recent finding reported that fath-1 knockdown results in a foraging defect [11]. However, it is not yet known whether or not these different functions of FATH-1 are interlinked. The molecular mechanisms underlying these functions are also unclear.

In this study, we systemically study the distribution and functions of FATH-1 and its catalytic products at the organismal, tissue, cellular and molecular level using $C$. elegans model. We establish the temporal and spatial expression patterns of FATH-1 in C. elegans. We confirm the overall phenotypes of fath-1 deficient animals and the tissues where FATH-1 performed most crucial functions. The subcellular defects caused by fath-1 inactivation are comprehensively investigated. We also identify the functional metabolites of FATH-1 by lipid analysis with mass spectrometry. Our studies reveal that FATH-1 and it metabolites function in a previously unappreciated, highly targeted and specific manner. Since $C$. elegans FATH-1 protein is highly homologous to mammalian FA2H enzyme [7], the results obtained from this would be of extensive reference value for related research fields.

\section{Materials and Methods}

C. elegans strains and maintenance

All C. elegans strains were maintained and handled at $20^{\circ} \mathrm{C}$ on nematode growth medium (NGM) as described previously [26]. Wildtype N2 strain and strains carrying transgenes zcIs17[ges-1::GFP(mit)],unc119(ed3)III;pwIs170[vha6p::GFP::rab-7+Cbr-unc-119(+)],rde-1(ne219)V; $\quad$ kbIs7[nhx-2p::rde-1+rol6(su1006))],pwIs216[vha-6p::mRFP::RME-1],rde-1(ne219)V;kzIs9[pKK1260(lin-26p::nls::GFP)+pKK1253(lin26p::rde-1)+rol-6(su1006)],unc-119(ed3)III;pwIs503[vha-6p::mans::GFP+Cbr-unc-119(+)] and hjIs37[vha- 


\section{Cellular Physiology Cell Physiol Biochem 2018;49:947-960 and Biochemistry Published \begin{tabular}{l|l} 
DOI: $10.1159 / 000493226$ & (c) 2018 The Author(s). Published by S. Karger AG, Basel
\end{tabular}}

Li et al.: FATH-1 Regulates Heptadecenoic Acid Production

6p::mRFP-PTS1+C.briggsae-unc-119(+)] were obtained from CGC (Caenorhabditis Genetics Center), which is funded by the NIH Office of Research Infrastructure Programs (P40 OD010440). ldrIs1 [dhs-3p::dhs3::GFP+unc-76(+)] was kindly provided by Dr. Pingsheng Liu (Institute of Biophysics, Chinese Academy of Sciences, Beijing). qxEx1867[vha-6p::mCHERRY::RAB-11], qxEx1317[ges-1p::GFP::RAB-11], qxIs110[ges$1 p:: m C H E R R Y:: R A B-5]$ were kindly provided by Dr. Xiaochen Wang (National Institute of Biological Sciences, Beijing). Other strains carrying transgenic arrays used in the study are as follows: sdaEx111[vha6p::GFP::TRAM-1+myo-2p::GFP], sdaEx105[fath-1p::GFP::fath-1+rol-6(su1006)].

\section{Molecular cloning and transgenesis}

fath-1 RNAi clone was constructed by inserting 437bp fragment containing exon 3 and exon 4 of fath-1 coding sequence into the HindIII site of vector L4440. To generate translational Pfath-1::GFP::fath-1 fusion, 4933bp promoter fragment of fath-1 was PCR amplified and inserted upstream of GFP, followed by the 2069bp fath-1 coding sequence inserted downstream of GFP into the pPD95.75 vector. To construct the intestinal ER marker Pvha-6::GFP::TRAM-1, 3300bp vha-6 promoter sequence and 1972bp TRAM-1 coding sequence were PCR amplified from N2 genomic DNA. The vha-6 promoter fragment and TRAM-1 fragment were then inserted upstream and downstream of GFP, respectively. All fragments obtained by PCR amplification were confirmed by sequencing before transgenesis. The primers used were as follows: for fath-1 RNAi, forward primer $5^{\prime}$ - AAA TCCGGAATGCTTTTCAA-3' and reverse primer 5'- TCCGTCCATCGGTGTTTTAT-3'; for fath-1 promoter, forward primer 5'- AGCGACTTCACСTCCTTTCT-3'and reverse primer 5'ATCCCCATATTTTCCACTGAA-3'; for fath-1 sequence, forward primer 5'- ATGGGGATAGGAGCAGATGA$3^{\prime}$ and reverse primer 5'-CACTCCCAAACCAGCATTTT $-3^{\prime}$; for vha-6 promoter, forward primer $5^{\prime}$ GGTTAAGCCGCAAGGAGG-3'and reverse primer $5^{\prime}$-TCTTCTTCGAATCGCTCTCC-3'; for TRAM sequence; forward primer 5' $5^{\prime}$-CCAACTCGGCAACGAAAT-3' and reverse primer 5'-TGGTAGGTTTTAGTCGCCCT-3'. Constructs used in this study were generated using ClonExpress TMOne Step Cloning Kit (Vazyme biotech). Pfath-1::GFP::fath-1 and Pvha-6::GFP::TRAM were injected into N2 at the concentration of 20ng/ $\mu$ l. pRF4(rol-6(su1006)) and Pmyo-2::GFP was used as co-injection markers, respectively [27].

\section{C. elegans development and life span assays}

For body length measurements, synchronized L1 larvae were placed on NGM agar plates with fath-1 RNAi bacteria or HT115 control and allowed to grow until control group reached young adult stage. Brightfield images of the worms on plates were taken using OLYMPUS SZX16 stereomicroscope fitted with a LEICA DFC310 camera. The body length of each individual was measured from the nose to the tail tip using ImageJ software. At least 40 individuals were examined per treatment group. The life-span assay was performed essentially as previously described [28]. At least 50 individuals were examined per treatment group.

\section{RNAi and fatty acid feeding}

fath-1 RNAi was performed by feeding synchronized L1 larvae with E.coli HT115 harboring the fath-1 RNAi clone. E.coli strain HT115 containing L4440 RNAi empty vector was used as negative control.

For diet supplementation, the fatty acid stock solutions were diluted to working concentration with $200 \mu \mathrm{L}$ OP50 overnight culture then added to the NGM plates. The plates were allowed to dry in the dark for 1-2 days at room temperature. Adult stage worms were transferred to these plates to lay eggs for 3 to 4 hours then removed. The effects of diet supplements were analyzed at the next generation. Fatty acids used for these studies include: $(R)-2-0 \mathrm{H}$ palmitic acid and $(S)-2-\mathrm{OH}$ palmitic acid, prepared as previously described as $7 \mathrm{mM}$ stocks in BSA [2]; $\Delta 10$-heptadecenoic acid and oleic acid (Sigma), prepared as $7 \mathrm{mM}$ stocks in BSA; alk 2-OH PA (custom-synthesized) and alk-PA (Cayman) with $\omega$-terminal alkyne, prepared as $10 \mathrm{mM}$ stocks in DMSO.

\section{Fatty acid labeling and dye-staining}

The "click chemistry" labeling was performed essentially as previously described [9]. Briefly, nematodes were soaked with alkynyl 2-OH-PA for 3 hours, washed once in M9 buffer, then fixed for 10 min with 3.7\% paraformaldehyde (Sigma) at room temperature. The worms were washed again with icecold PBS and permeabilized with pre-cooled $\mathrm{MeOH}$ for $10 \mathrm{~min}$ at $-20^{\circ} \mathrm{C}$. The worms were then blocked with PBSTB $(0.1 \%$ Tween-20, $20 \mathrm{mg} / \mathrm{mL}$ BSA in PBS) for $30 \mathrm{~min}$ at room temperature followed by three times washing with PBS, 5 min each. The labeling was performed by soaking the worms with freshly mixed 


\section{Cellular Physiology Cell Physiol Biochem 2018;49:947-960

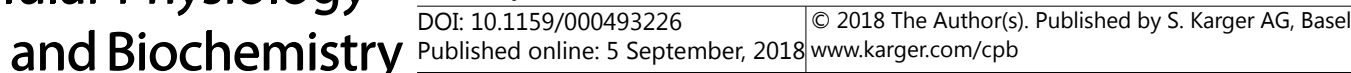

Li et al.: FATH-1 Regulates Heptadecenoic Acid Production

click chemistry reaction solution [20 $\mu$ M Cy3-azide (Life technology), $10 \mathrm{mM}$ TECP (Sigma), $100 \mu \mathrm{M}$ TBTA (Sigma), $1 \mathrm{mM} \mathrm{CuSO4} \bullet 5 \mathrm{H} 2 \mathrm{O}, 0.1 \%$ Tween-20 in PBS] for 1 hour at room temperature. The samples were then washed 3 times (5 min each) with 1\% Tween-20, 0.5 mM EDTA in PBS and then two times with PBS alone before confocal imaging.

To label lipid droplets in fixed worms, young adult hermaphrodites were soaked with $1 \mu \mathrm{g} / \mathrm{mL}$ BODIPY 493/503 (Life technologies) following a previously described staining method [15]. To examine the lipid uptake process, $1 \mu \mathrm{M}$ fluorescent fatty acid analog BODIPY 500/510 (Life technologies) was fed to young adults on plates as described previously [17]. Intestinal lysosomes were labeled by $1 \mu \mathrm{M}$ LysoTracker Green DND-26 (Invitrogen) added to the NGM plates [29].

\section{Microscopic imaging}

For confocal imaging, live worms were mounted on $2 \%$ agarose pads on a microscopic slide and immobilized with $0.5 \mathrm{mM}$ levamisole [30]. Fluorescent images were captured using Nikon A1 confocal microscope with 488, 543 lasers and 63x oil immersion objective. GFP, Lysotracker Green, BODIPY 493/503, BODIPY 500/510 were excited by $488 \mathrm{~nm}$ laser; mCherry, RFP and Cy3 were excited by $543 \mathrm{~nm}$ laser. Brightfield images of worms on the plates were acquired under OLYMPUS SZX16 stereomicroscope fitted with a LEICA DFC310 camera. The endosome aggregates and the numbers of droplets were scored within a unit area of $200 \mu \mathrm{m}^{2}$ using Image J in a double-blind fashion. At least five different areas were chosen and quantified in each animal, and 8 animals were scored for each experimental group.

Fatty acid analysis with mass spectrometry

Total lipids were extracted from C. elegans samples by a modified Bligh and Dyer procedure and FA methyl esters were prepared as previously described [31]. The free fatty acids were released from methyl esters by incubation with $1 \mathrm{M} \mathrm{KOH}$ solution in methanol. The charge-switch derivatization was performed using $N$-(4-1'-aminoethylphenyl) pyridinium (AEPP) which was synthesized as described [32]. Mass spectrometric analysis of AEPP-derivatized FAs was performed using a TSQ Vantage mass spectrometer (Thermo Fisher Scientific, San Jose, CA) equipped with an electrospray ion source. Product ion scanning or precursor ion scanning was performed in the positive-ion mode as previously described [32].

\section{Statistical analysis}

For body lengths and image analyses, statistical analysis was performed with unpaired, two-tailed Student's t test using Prism 5.0 software (GraphPad). Significance was accepted for $\mathrm{p}<0.05$.

\section{Results}

C. elegans FATH-1 is expressed in multiple developmental stages and in multiple tissues

To determine the spatio-temporal expression patterns of FATH-1 during C. elegans development, we constructed a transgene expressing GFP::FATH-1 under the control of fath-1 endogenous promoter (Fig. 1A). Translational FATH-1 reporter was detected in most developmental stages including embryos, L1, L4 larvae and adults (Fig. 1B-F). Strong FATH1 expression was observed in multiple tissue types such as neuronal cells, intestinal cells, and epidermal cells. In contrast, no GFP signal was detected in the gonad or muscle cells. Close-up images of the intestinal cells revealed that most FATH-1 proteins were uniformly distributed in the cytoplasm, with moderate enrichment in the nuclei (Fig. 1B-1F). These results suggest that the functions of FATH-1 are involved in multiple developmental stages and in multiple tissues.

\section{Loss of fath-1 function affects C. elegans growth and lifespan}

To study the functions of FATH-1 in C. elegans, we first documented the overall phenotypes of fath-1 deficient animals. To avoid the problem of early lethality, we inactivated fath-1 gene expression by RNAi feeding in the wildtype background from L1-larval stage. Bright-field images and body-length measurement results showed that worm growth was significantly inhibited after fath-1 knockdown (Fig. 2A, B). To evaluate whether loss of fath-1 function affects the lifespan of C. elegans, we performed survival curve assay after inactivation of fath-1 
Fig. 1. The spatial-temporal distribution patterns of FATH-1 in C. elegans. (A) A diagram shows the FATH-1 protein domains, including four transmembrane domains and one Cyt-b5 domain. GFP was inserted at the N-terminus of FATH-1. (B-F) The distribution patterns of fusion protein GFP::FATH-1 in the $C$. elegans at embryonic (B), L1 larvae (C), L4 larvae (D) and adult (E, F) stage. (D) is a composite confocal image showing GFP::FATH-1 localization in the epidermal cells. (E-E") shows the GFP::FATH-1 distribution in the intestine (solid arrowhead) and nervous system (open arrowhead). (F-F") shows the GFP::FATH-1 distribution in the intestine (solid arrowhead) but not the gonad (open arrow). Scale bar in D, $20 \mu \mathrm{m}$; Scale bars in B,C,E and F, $10 \mu \mathrm{m}$.

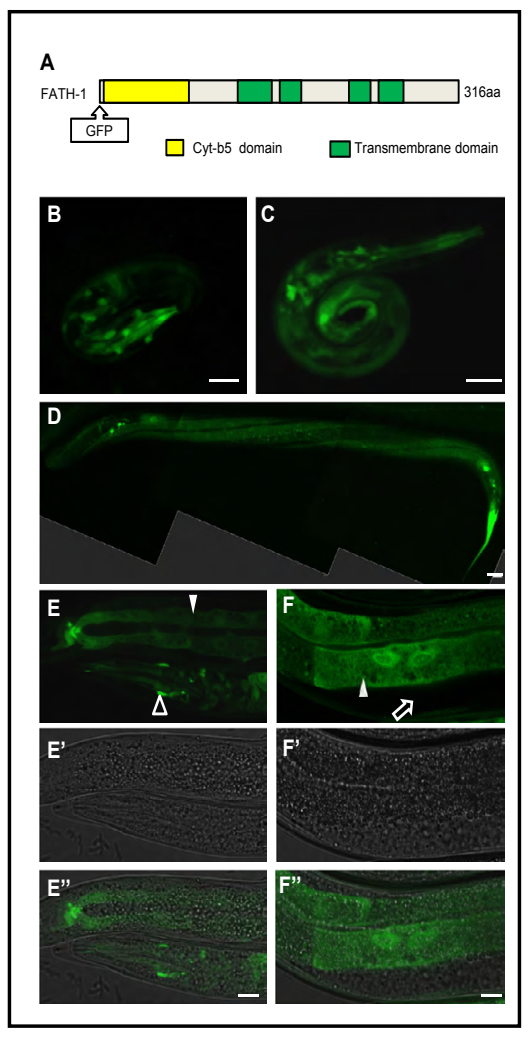

Fig. 2. The systemic and tissue-specific effect of fath-1 inactivation. (A) Bright-field images of worms fed with HT115 control or fath-1 RNAi bacteria from L1 to young adult stage. Scale bar, $400 \mu \mathrm{m}$. (B) Changes of body lengths of worms treated with HT115 or fath-1 RNAi from L1 stage. $N \geq 45$ for each data set. (C) Survival curves of the worms treated with HT115 or fath-1 RNAi from L1. $\mathrm{N} \geq 75$ for each data set. (D) Survival curves of dauer-like animals caused by starvation (control) or by fath-1 RNAi treatment. $\mathrm{N} \geq 81$ for each data set. (E) Body length measurements of wildtype, epidermisspecific RNAi (epi KD) and intestine-specific RNAi (int KD) worms treated with fath-1 RNAi, dpy-7 RNAi (positive control for epidermalspecific RNAi) or HT115 (negative control). N $\geq 52$ for each data set.

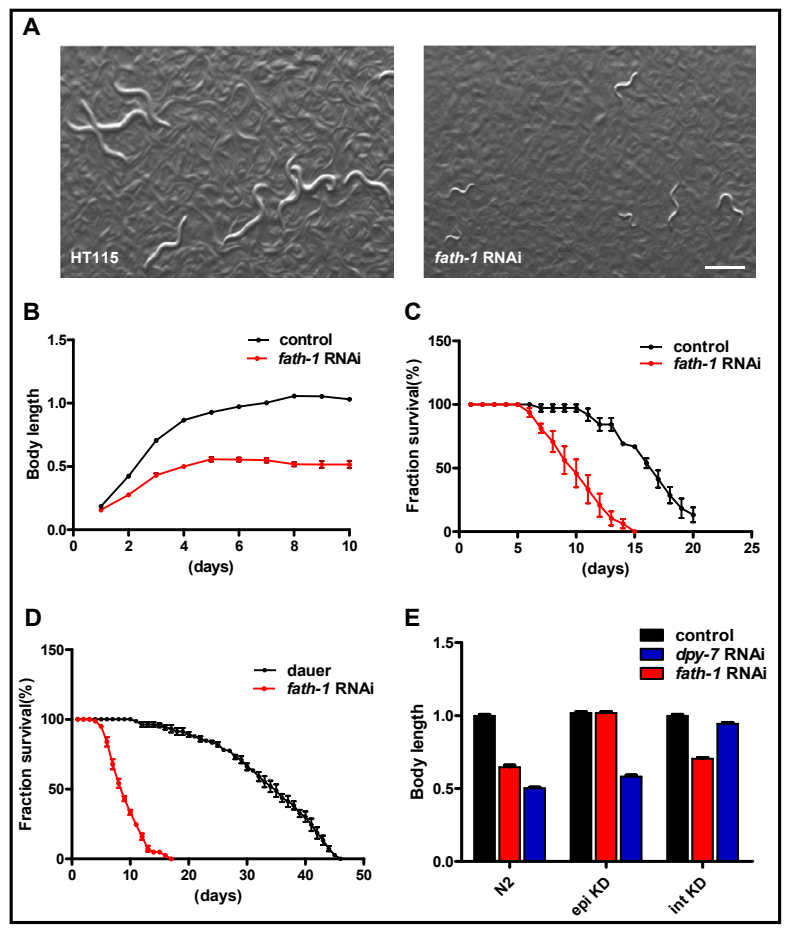

expression. As shown in Fig. 2C, animals treated with fath-1 RNAi displayed greatly shortened lifespan compared with control groups. Consistent with previous reports, we observed a small portion of individuals with dauer-like morphology in the population of fath-1 deficient animals $[6,8]$. Yet they do not exhibit extended lifespan like the wildtype dauer controls (Fig. 
2D). Together, these results reveal that FATH-1 function is essential for maintaining normal development and survival.

Since FATH-1 is present in multiple tissue types, we next performed tissuespecific RNAi experiments to find out in which tissue FATH1 plays a more crucial role. We utilized strains designated for epidermis-specific RNAi (epi KD) and intestine-specific RNAi (int KD) as previously reported [12]. When fath1 was inactivated only in the intestine, it affected the worm growth in a similar degree as when systemic knockdown was applied. In comparison, loss of fath-1 function in the epidermis displayed no obvious effect on development (Fig. 2E). These observations indicate that FATH-1 maintains normal growth and development mainly by functioning in the intestinal cells.

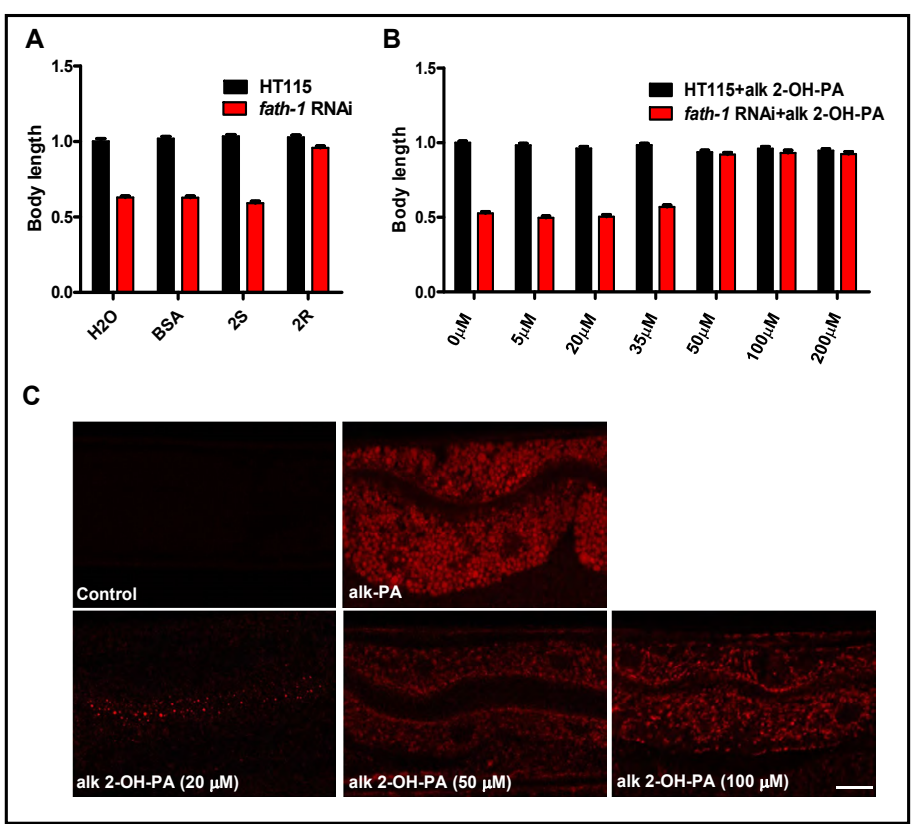

Fig. 3. The stereospecificity and subcellular localization pattern of 2-OH FAs. (A) Body length measurement of fath-1 RNAi worms rescued by BSA control, $350 \mu \mathrm{M}(R)-2-0 H$ FA $(2 \mathrm{R})$ or $350 \mu \mathrm{M}(S)$ 2-OH FA (2S) supplementation. $\mathrm{N} \geq 50$ for each data set. (B) Body length measurements showing the rescuing effects of $5,20,35,50$, 100 or $200 \mu \mathrm{M}$ alkynyl 2-OH-PA supplementation on the growth arrest defect caused by fath-1 RNAi. $\mathrm{N} \geq 50$ for each data set. (C) Distribution of fatty acids and derivatives in the C. elegans intestine labeled by "Click chemistry" approach. PA, palmitic acid; OH-PA, 2-hydroxy plamitic acid; alk, alkynyl; az, azide. Scale bar, $5 \mu \mathrm{m}$.

\section{The FATH-1 catalytic}

product 2-OH FA has stereospecificity and spatially restricted subcellular distribution pattern

FATH-1 is predicted to function primarily by catalyzing the hydroxylation of FA at the C-2 position to produce 2-hydroxy FAs (2-OH FAs) [13]. To test this hypothesis, we performed rescue experiment by supplementing fath-1 deficient animals with 2-hydroxy palmitic acid (2-OH PA). It was previously reported that the mammalian FA2H is stereospecific for the production of the 2-OH FA enantiomer with $\mathrm{R}$ conformation $((R)-2-\mathrm{OH})$ [2]. Therefore, both $(R)$-2-OH FA and $(S)$-2-OH FA enantiomers were included in the rescuing analysis. The results showed that $(R)-2-\mathrm{OH}$ FA supplement nicely rescued the growth defect caused by fath-1 RNAi, while (S)-2-OH FA did not (Fig. 3A). These observations suggested that FATH1 primarily function through its catalytic product $(R)-2-0 H$ FAs in $C$. elegans, similar as its mammalian counterpart.

At the subcellular level, we examined in which cellular compartments 2-OH FA and its derivatives are localized. To visualize 2-OH FA in vivo at the subcellular resolution, we performed a chemical labeling approach called "click chemistry" as reported by Charron et al. [14]. Adult C. elegans were fed with 2-OH palmitic acid alkyne (alk 2-OH PA), then fixed and subjected to click chemistry reaction with azide-Cy3. To ensure the functionality of alkyne-tagged OH-PA molecules and to determine a suitable concentration for alk 2-OH PA supplementation, we performed a rescuing experiment with different titrations of alk 2-OH PA ranging from $5 \mu \mathrm{M}$ to $200 \mu \mathrm{M}$. The results showed that a supplementation of $50 \mu \mathrm{M}$ alk 2-OH PA was sufficient to rescue the growth defect of fath-1 knockdown animals (Fig. 3B). It confirms that the alkynyl group does not affect the functionality of OH-PA molecules. We 
then examined OH-PA distribution within the intestinal cell using "click chemistry" labeling method, with non-hydroxylated palmitic acid alkyne (alk-PA) as control. As shown in Fig. 3B, non-hydroxylated alk-PA exhibited uniform subcellular distribution pattern, whereas hydroxylated alk 2-OH PA are mostly accumulated in membrane-like organelles. In addition, worms supplied with smaller amounts of alk 2-OH PA $(20 \mu \mathrm{M})$ displayed signal enrichment at the apical side of the intestinal cells, suggesting that the hydroxylated fatty acids may preferentially distribute apically within polarized cells (Fig. 3C). Collectively, these data indicated that the primary FATH-1 metabolites 2-OH FA are likely preferentially enriched in membrane-bound cellular organelles in the intestinal cells.

\section{fath-1 deficiency affects lipid droplets, peroxisomes and endosomes}

Our next question is how FATH-1 function affects subcellular structures and processes of the intestinal cells. We specifically looked into the membrane-bound organelles, namely endocytic vesicles, endoplasmic reticulum, Golgi apparatus, mitochondria, lipid droplets, peroxisomes and lysosomes (Fig. 4). Firstly, we explored the changes of lipid storage in the intestinal cells. BODIPY 493/503 staining or DHS-3::GFP labeling showed that the number of lipid droplets was significantly reduced when fath-1 was inactivated (Fig. 4A-C) $[15,16]$. However, the lipid uptake process appeared not affected by fath-1 knockdown, as demonstrated by BODIPY 500/510 feeding of live worms (Fig. 4A) [17]. It indicates that FATH-1 might be involved in lipid droplets formation or maintenance rather than the lipid uptake process. Consistently, the peroxisomes which are essential for fatty acid metabolism also formed abnormal aggregations within the fath-1 depleted intestine, as shown by mRFP::PTS1 (peroxisome targeting signal 1) labeling (Fig. 4A, D).

We next examined whether loss of FATH-1 function affect endosomes. The marker proteins include RAB- 5 for early endosomes, RAB-7 for late endosomes, RAB- 11 for apical recycling endosomes and RME-1 for basolateral recycling endosomes $[18,19]$. The results showed that among the endocytic vesicles tested, the distribution of RAB-7-labeled late endosomes and RAB-11-labeled apical recycling endosomes was particularly affected by loss of fath-1. RAB-7 and RAB-11 both formed large aggregates at the apical region of intestinal cell after fath-1 knockdown, while other types of endosomes remained undisturbed (Fig. 4A, E, F). Together, these results suggest that loss of FATH-1 function specifically affects apical membrane trafficking, which is in accord with the apical preference of OH-FA distribution within the intestinal cells and previous findings (Fig. 3C) [9].

Unlike lipid droplets, peroxisomes and endosomes, most other membrane-bound organelles such as endoplasmic reticulum (TRAM::GFP), Golgi apparatus (MANS::GFP), mitochondria (GES-1::GFP) and lysosomes (lysotracker) were not significantly affected by loss of FATH-1 function [19, 20] (Fig. 4A). To summarize, these data suggest that FATH-1 function is important for the formation and maintenance of endosomes, peroxisomes and lipid droplets, which is likely the cause of disturbed growth and development of fath-1 deficient nematodes.

\section{FATH-1 function in C. elegans intestine is mediated by heptadecenoic acid}

At the molecular level, we sought to identify the key functional metabolites of FATH-1. We compared the FA profile as regulated by fath-1 RNAi and examined whether this regulation could bereversed by $(R)$-2-OH PA. FAswere analyzed as $N$-(4-1'-aminoethylphenyl) pyridinium derivatives $\left([\mathrm{FA}+\mathrm{AEPP}]^{+}\right)$by mass spectrometry analysis. Surprisingly, the results showed that the amounts of most major FAs were not affected by either fath-1 RNAi or $(R)-2-\mathrm{OH}$ PA treatment. However, loss of FATH-1 function significantly decreased the relative percentage of $\mathrm{C} 17: 1(\mathrm{~m} / \mathrm{z} 494)$ in total FAs (Fig. 5A, 5B) and this decrease was reversed by $(R)-2-0 \mathrm{H}$ PA supplement (Fig. 5C, 6A). The relative content of C17:0 was also mildly affected by fath1 deficiency. Product-ion MS spectral analysis of $\mathrm{m} / \mathrm{z} 494$ ion by ESI-MS/MS (Electrospray Ionization Mass Spectrometer) demonstrated informative fragment ions for double bond determination of C17:1, which identified it as $\Delta 10 \mathrm{C} 17: 1$ (heptadecenoic acid, Fig. 5D, E). We next examined the involvement of FATH-1-regulated $\triangle 10 \mathrm{C} 17: 1$ in growth and development.

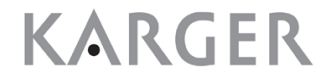




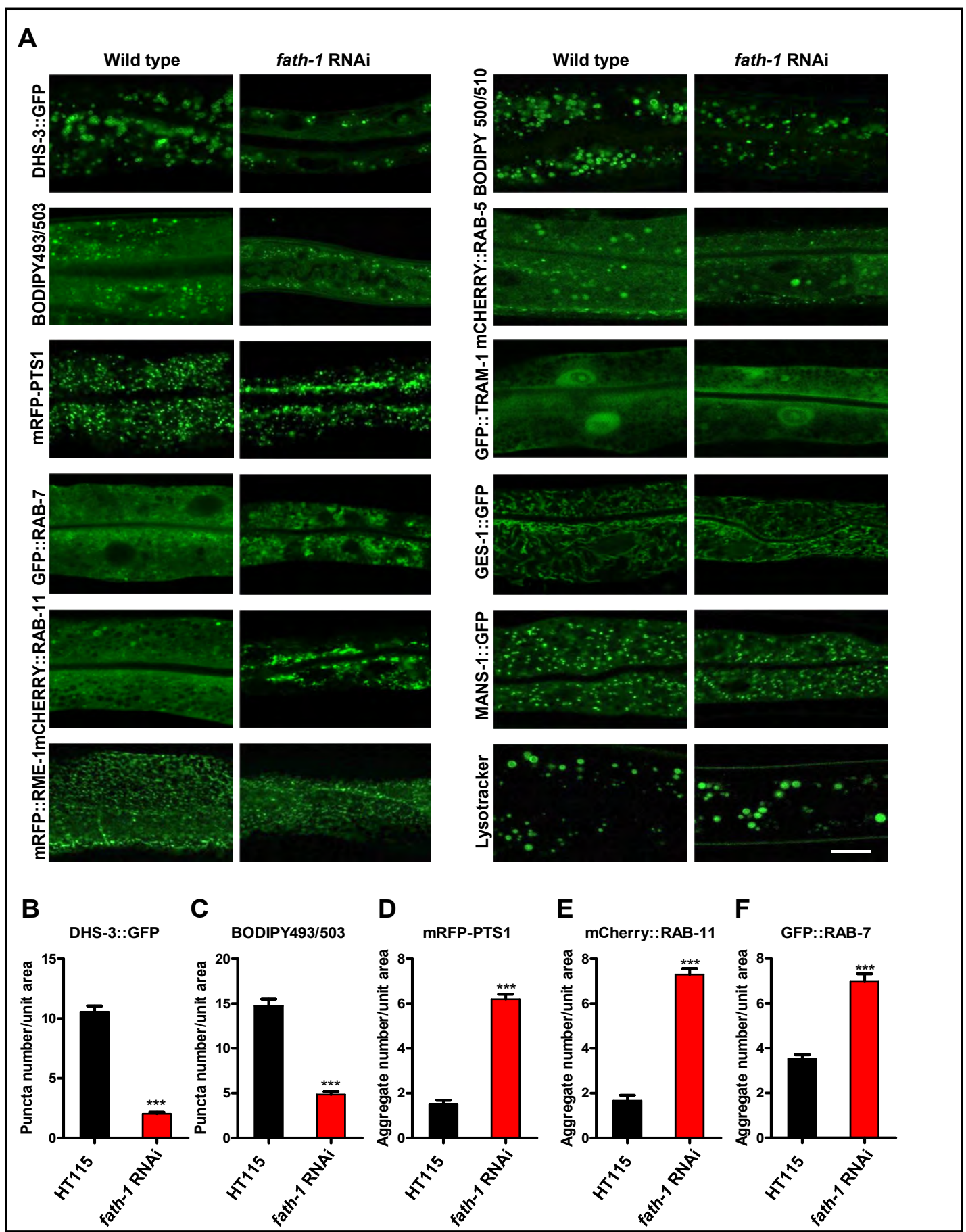

Fig. 4. The subcellular defects caused by fath-1 inactivation. (A) Representative fluorescent confocal images showing intestinal cellular organelles of young adult $C$. elegans treated with HT115 or fath-1 RNAi. The organelles include: lipid droplets (DHS-3::GFP, BODIPY 493/503); peroxisomes (mRFP::PTS1); late endosomes (GFP::RAB-7); apical recycling endosomes (mCherry::RAB-11); basolateral recycling endosomes (mRFP::RME-1); early endosomes (mCherry::RAB-5); endoplasmic reticulum (GFP::TRAM-1); mitochondria (GES-1::GFP) or Golgi (MANS-1::GFP). Lysosomes were marked by lysotracker green. BODIPY 500/510 visualizes the process of fatty acid uptake. The scale bar represents $10 \mu \mathrm{m}$. (B, C, D, E, F) Quantification of the numbers of endosome and peroxisome aggregates or lipid droplets in worms lacking fath-1 expression, corresponding to results shown in $\mathrm{A}$. $\mathrm{N} \geq 40$ for each data set. Error bars, $\pm \mathrm{SEM}$; ${ }^{* * *} \mathrm{p}<0.001$. 


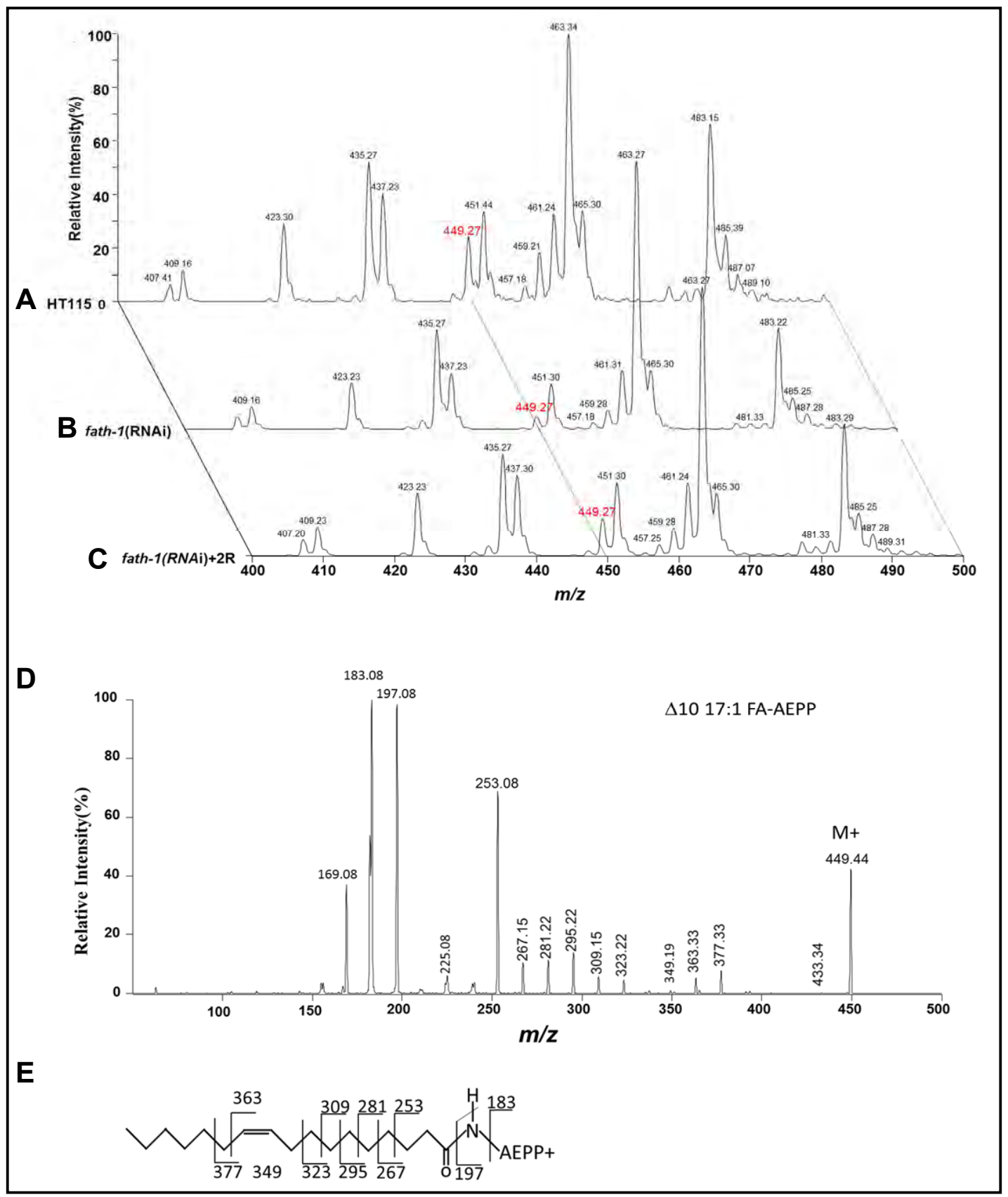

Fig. 5. The fatty acid profiles affected by fath-1 inactivation. (A-C) Representative mass spectra of AEPP derivatives of total FAs from wildtype animals fed with HT115 control (A), fath-1 RNAi (B) or fath-1 RNAi plus $350 \mu \mathrm{M}(R)-2-\mathrm{OH}$ FA (2R) supplementation (C). (D) Representative product-ion MS spectrum of $m / z$ 494 ion by ESI-MS/MS. (E) The structure of $\triangle 10 \mathrm{C} 17: 1$ AEPP derivative and fragment ion peak assignment.

In wild type animals with a normal level of $\Delta 10 \mathrm{C} 17: 1$, treatment of either $\Delta 10 \mathrm{C} 17: 1$ or $\mathrm{C} 18: 1$ had no effect on body length. However, the decreased body length by loss of FATH-1 function was totally reversed by $\Delta 10 \mathrm{C} 17: 1$, but not by C18:1 (Fig. 6B, C). Likewise, supplementation of $\triangle 10 \mathrm{C} 17: 1$ rescued fully the defects of lipid droplet formation, and partially the defects of peroxisome and endosome aggregation (Fig. 7A-E). Taken together, these data suggest that $\triangle 10 \mathrm{C} 17: 1$ is a major downstream effector mediating the physiological roles of FATH-1. 


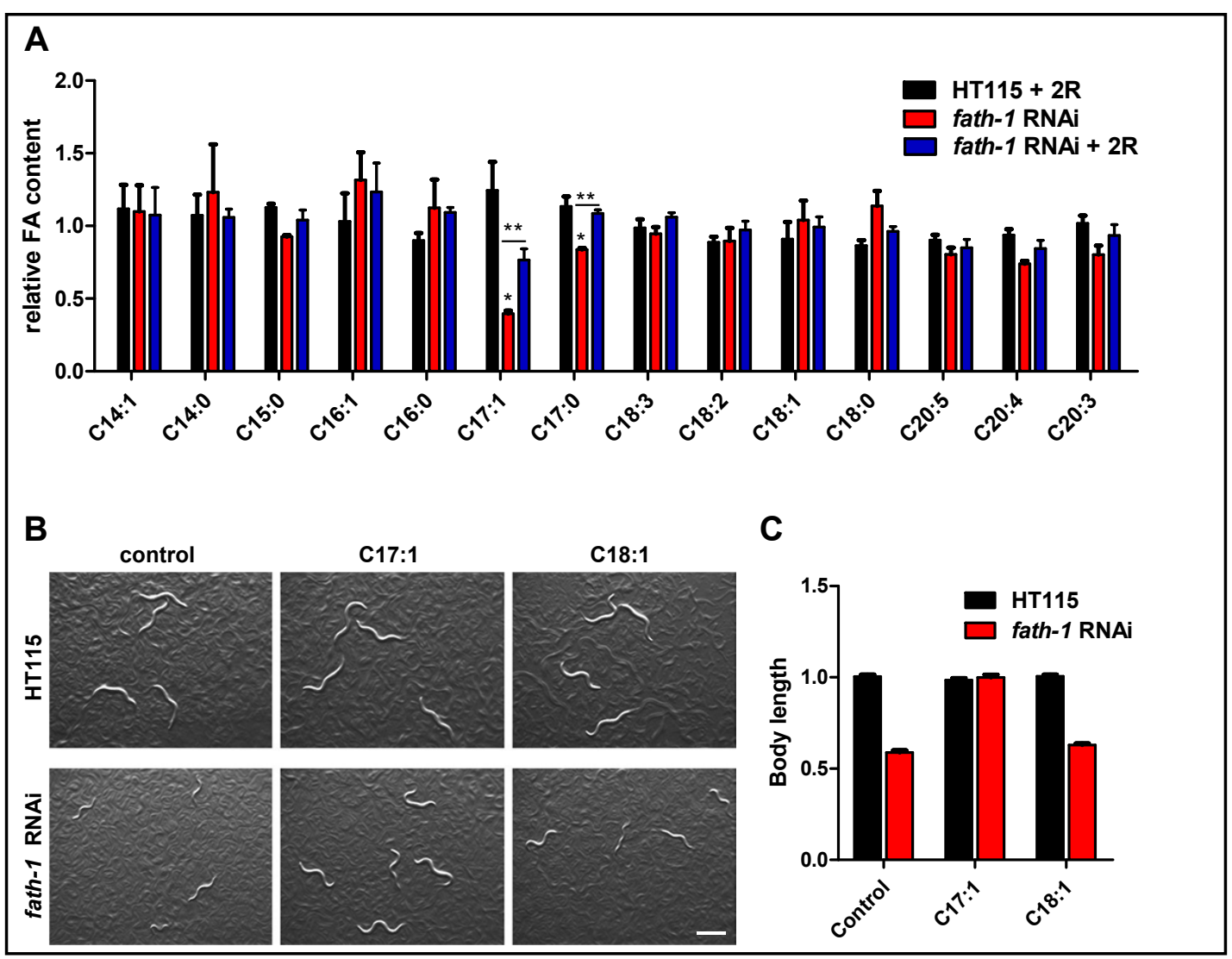

Fig. 6. $\triangle 10 \mathrm{C} 17: 1$ mediates FATH-1 function in growth and development. (A) Relative FA abundance normalized to HT115 control group. (three biological replicates, $\mathrm{N}>1000$ /condition). Error bars, \pm SEM; ${ }^{*} \mathrm{p}<0.05,{ }^{* *} \mathrm{p}<0.01$. (B) Bright-field images of worms fed with HT115 control or fath-1 RNAi treated with BSA, $\Delta 10 \mathrm{C} 17: 1$ or $\Delta 9 \mathrm{C} 18: 1$. Scale bar, $400 \mu \mathrm{m}$. (C) Body length measurement of worms fed with HT115 control or fath-1 RNAi treated with BSA, $\Delta 10 \mathrm{C} 17: 1$ or $\Delta 9 \mathrm{C} 18: 1$. N $\geq 51$ for each data set. 


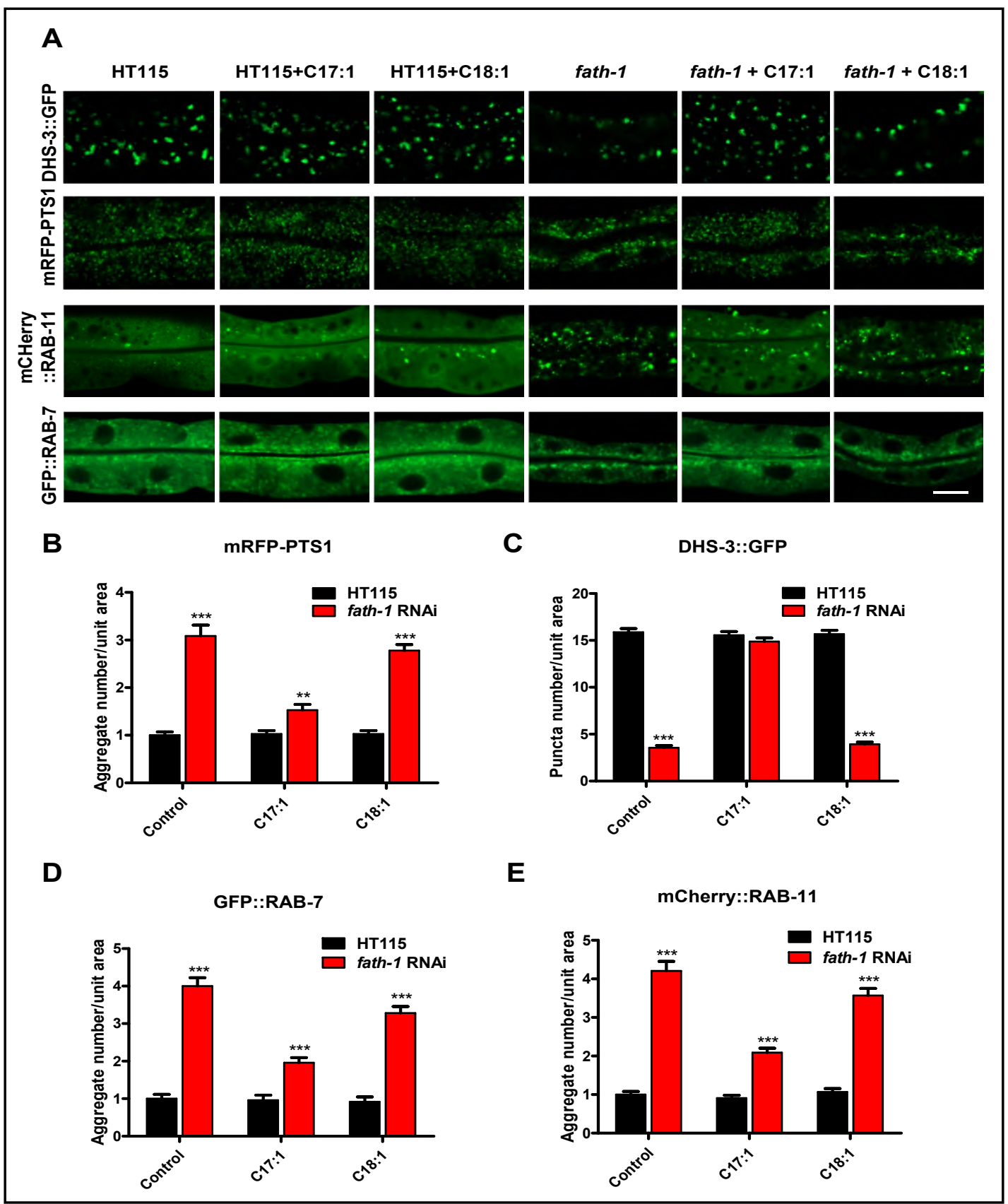

Fig. 7. $\Delta 10 \mathrm{C} 17: 1$ rescues subcellular defects of fath-1 deficient worms (A) Representative fluorescent confocal images showing intestinal cellular organelles of $C$. elegans treated with HT115, HT115 plus 100 $\mu \mathrm{M} \Delta 10 \mathrm{C} 17: 1$, HT115 plus 100 $\mu \mathrm{M} \Delta$ 9C18:1, fath-1 RNAi, fath-1 RNAi plus $100 \mu \mathrm{M} \Delta 10 \mathrm{C} 17: 1$ or fath-1 RNAi plus $100 \mu \mathrm{M} \Delta$ 9C18:1. The organelles include: lipid droplets (DHS-3::GFP); peroxisomes (mRFP::PTS1); late endosome (GFP::RAB-7) and apical recycling endosome (mCherry::RAB-11). The scale bar represents 10 $\mu \mathrm{m}$. (B, C, D, E) Quantification of the numbers of endosome and peroxisomes aggregates or lipid droplets in worms fed with HT115 or fath-1 RNAi treated with $\Delta 10 \mathrm{C} 17: 1$ or $\Delta 9 \mathrm{C} 18: 1$, corresponding to results shown in $\mathrm{A} . \mathrm{N}=20$ for each data set. Error bars, $\pm \mathrm{SEM}{ }^{* *} \mathrm{p}<0.01,{ }^{* * *} \mathrm{p}<0.001$. 


\section{Cellular Physiology Cell Physiol Biochem 2018;49:947-960

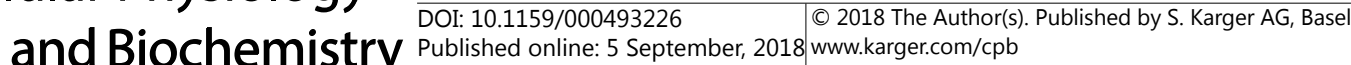

Li et al.: FATH-1 Regulates Heptadecenoic Acid Production

\section{Discussion}

In this work, we systemically investigate the distribution and functions of fatty acid 2-hydroxylase and its catalytic products using C. elegans as the model. We find that C. elegans FATH-1 is important for normal development and survival of the nematodes. Such functions are achieved mainly by regulating the dynamics of endosomes, peroxisomes and lipid droplets in the intestine. $C$. elegans intestinal cell has been used as a popular model for monitoring structures and processes at subcellular resolution under in vivo physiological conditions in live animals. Conclusions obtained from studying FATH-1 function in C. elegans intestinal cells is likely applicable to other cell types as well. Previous reports regarding human FA2H mostly involve neurodegenerative disorders, especially HSP. Interestingly, HSP is believed to be caused partially by defects in transport of proteins and lipids in motor neurons, which is in accordance with FATH-1 function in C. elegans intestinal cells [21]. Furthermore, previous study documented that FATH-1 is required for normal foraging behavior, which involves a well-characterized circuit of sensory neurons, interneurons and motor neurons [11,22]. Our translational reporter analysis also showed that FATH-1 is expressed in multiple neurons in the head and tail regions, indicating that loss of FATH-1 function could also directly impair the function of neuronal cells. Therefore, more detailed investigation using C. elegans model may help elucidate the contribution of FA2H in the pathogenesis of HSP.

C. elegans FATH-1 was previously reported to be involved in apical polarity establishment of the intestinal cells [9]. Our observation that the FATH-1 metabolites preferentially accumulates at the apical compartment of the intestine, and that loss of FATH-1 specifically affects apical protein trafficking may help explain the tubular polarity defect caused by FATH-1 deficiency. In addition, fath-1 was identified as a positive regulator of intestinal innate immune response [10]. The fact that FATH-1 function particularly affects the apical compartment of the intestinal cells may shed new light on the investigation of the underlying mechanisms of its immune-regulating roles, since the apical side of the intestine serves as the frontline barrier against invading pathogens.

In this work, we also identified heptadecenoic acid (C17:1) as a downstream target of C. elegans FATH-1. The previous knowledge about the physiological roles of heptadecenoic acid is very limited. Heptadecenoic acid has been recognized as a minor constituent of ruminant fats and used as a potential marker of microbial biomass [23, 24]. It was assumed that the rumen microbial originated odd chain fatty acids are exported to milk fat after intestinal absorption. Acetonitrile chemical ionization-MS/MS fragmentation in conjunction with GC resolution of $\mathrm{C} 17: 1$ isomers demonstrated that $\Delta 9 \mathrm{C} 17: 1$ is the overwhelming heptadecenoic isomer in ruminant milk and intramuscular fat, while $\Delta 10 \mathrm{C} 17: 1$ is virtually absent [25]. Although the C17:1 standard available from commercial chemical companies is the $\Delta 10$ isomer, no reports on the origin or functions of $\Delta 10 \mathrm{C} 17: 1$ have been found. Enzymes responsible for the generation of $\Delta 10 \mathrm{C} 17: 1$ in C. elegans and the metabolic mechanisms leading to its unique function in development require extensive future investigation. Nevertheless, our discovery brings heptadecenoic acid out into the spotlight and opens new possibilities of therapeutical approaches for treating FA2H-related diseases.

\section{Conclusion}

We show in this study the functions of FA2H at the organismal, tissue, cellular and molecular level using C. elegans as the model. We demonstrate that FATH-1 is essential for maintaining normal development and survival, most crucially required in the intestine, specifically affects lipid droplets, peroxisomes and endosomes of the intestinal cells, and partially works through heptadecenoic acid to carry out its functions. These findings reveal a previously unappreciated specific manner of FATH-1 function, and may help understand the cellular mechanisms of the FA2H-related processes across species. 


\section{Cellular Physiology Cell Physiol Biochem 2018;49:947-960

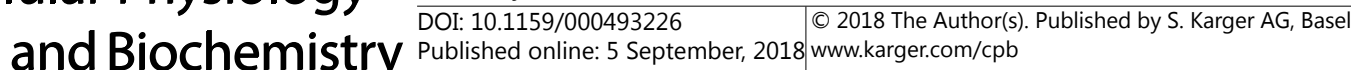

\section{Acknowledgements}

The authors would like to thank Xiaochen Wang, Pingsheng Liu and the Caenorhabditis Genetics Center for reagents. This work is supported by National Natural Science Foundation of China (31471265, 31670912, 31371437, 31570806 and 31620103906), National Institutes of Health Grant DK097608, the Natural Science Foundation of Jiangsu Province of China (BK20150006 and BK20160009), the Six Talent Peaks Project of Jiangsu Province (2015-SWYY-024 and 2015-SWYY-025), Jiangsu Provincial Innovative Research Team, Program for Changjiang Scholars and Innovative Research Team in University (IRT1075) and the Priority Academic Program Development of Jiangsu Higher Education Institutions. Some strains were provided by CGC (Caenorhabditis Genetics Center), which is funded by the NIH Office of Research Infrastructure Programs (P40 OD010440).

\section{Disclosure Statement}

The authors declare that there are no conflicts of interest regarding the publication of this paper.

\section{References}

1 Ibarguren M, Lopez DJ, Escriba PV: The effect of natural and synthetic fatty acids on membrane structure, microdomain organization, cellular functions and human health. Biochim Biophys Acta 2014;1838:15181528.

-2 Guo L, Zhang X, Zhou D, Okunade AL, Su X: Stereospecificity of fatty acid 2-hydroxylase and differential functions of 2-hydroxy fatty acid enantiomers. J Lipid Res 2012;53:1327-1335.

3 Guo L, Zhou D, Pryse KM, Okunade AL, Su X: Fatty acid 2-hydroxylase mediates diffusional mobility of Raftassociated lipids, GLUT4 level, and lipogenesis in 3T3-L1 adipocytes. J Biol Chem 2010;285:25438-25447.

4 Kota V, Hama H: 2'-Hydroxy ceramide in membrane homeostasis and cell signaling. Adv Biol Regul 2014;54:223-230.

5 Edvardson S, Hama H, Shaag A, Gomori JM, Berger I, Soffer D, Korman SH, Taustein I, Saada A, Elpeleg O: Mutations in the fatty acid 2-hydroxylase gene are associated with leukodystrophy with spastic paraparesis and dystonia. Am J Hum Genet 2008;83:643-648.

6 Zhu H, Shen H, Sewell AK, Kniazeva M, Han M: A novel sphingolipid-TORC1 pathway critically promotes postembryonic development in Caenorhabditis elegans. Elife 2013;2:e00429.

7 Eckhardt M, Yaghootfam A, Fewou SN, Zoller I, Gieselmann V: A mammalian fatty acid hydroxylase responsible for the formation of alpha-hydroxylated galactosylceramide in myelin. Biochem J 2005;388:245-254.

8 Tewari M, Hu PJ, Ahn JS, Ayivi-Guedehoussou N, Vidalain PO, Li S, Milstein S, Armstrong CM, Boxem M, Butler MD, Busiguina S, Rual JF, Ibarrola N, Chaklos ST, Bertin N, Vaglio P, Edgley ML, King KV, Albert PS, Vandenhaute J, et al.: Systematic interactome mapping and genetic perturbation analysis of a C. elegans TGF-beta signaling network. Mol Cell 2004;13:469-482.

-9 Zhang H, Abraham N, Khan LA, Hall DH, Fleming JT, Gobel V: Apicobasal domain identities of expanding tubular membranes depend on glycosphingolipid biosynthesis. Nat Cell Biol 2011;13:1189-1201.

10 Pukkila-Worley R, Feinbaum RL, McEwan DL, Conery AL, Ausubel FM: The evolutionarily conserved mediator subunit MDT-15/MED15 links protective innate immune responses and xenobiotic detoxification. PLoS Pathog 2014;10:e1004143.

11 Zhu H, Sewell AK, Han M: Intestinal apical polarity mediates regulation of TORC1 by glucosylceramide in C. elegans. Genes Dev 2015;29:1218-1223.

12 Qadota H, Inoue M, Hikita T, Koppen M, Hardin JD, Amano M, Moerman DG, Kaibuchi K: Establishment of a tissue-specific RNAi system in C. elegans. Gene 2007;400:166-173.

$\checkmark 13$ Hama H: Fatty acid 2-Hydroxylation in mammalian sphingolipid biology. Biochim Biophys Acta 2010;1801:405-414. 


\section{Cellular Physiology Cell Physiol Biochem 2018:49:947-960

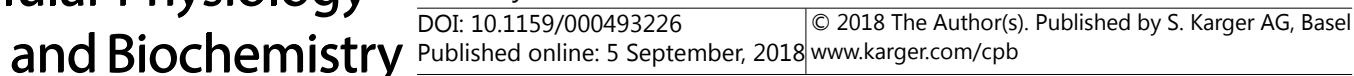

Li et al.: FATH-1 Regulates Heptadecenoic Acid Production

14 Charron G, Zhang MM, Yount JS, Wilson J, Raghavan AS, Shamir E, Hang HC: Robust fluorescent detection of protein fatty-acylation with chemical reporters. J Am Chem Soc 2009;131:4967-4975.

-15 Klapper M, Ehmke M, Palgunow D, Bohme M, Matthaus C, Bergner G, Dietzek B, Popp J, Doring F: Fluorescence-based fixative and vital staining of lipid droplets in Caenorhabditis elegans reveal fat stores using microscopy and flow cytometry approaches. J Lipid Res 2011;52:1281-1293.

16 Zhang P, Na H, Liu Z, Zhang S, Xue P, Chen Y, Pu J, Peng G, Huang X, Yang F, Xie Z, Xu T, Xu P, Ou G, Zhang SO, Liu P: Proteomic study and marker protein identification of Caenorhabditis elegans lipid droplets. Mol Cell Proteomics 2012;11:317-328.

-17 Soukas AA, Kane EA, Carr CE, Melo JA, Ruvkun G: Rictor/TORC2 regulates fat metabolism, feeding, growth, and life span in Caenorhabditis elegans. Genes Dev 2009;23:496-511.

18 Pant S, Sharma M, Patel K, Caplan S, Carr CM, Grant BD: AMPH-1/Amphiphysin/Bin1 functions with RME1/Ehd1 in endocytic recycling. Nat Cell Biol 2009;11:1399-1410.

19 Chen B, Jiang Y, Zeng S, Yan J, Li X, Zhang Y, Zou W, Wang X: Endocytic sorting and recycling require membrane phosphatidylserine asymmetry maintained by TAT-1/CHAT-1. PLoS Genet 2010;6:e1001235.

-20 Rolls MM, Hall DH, Victor M, Stelzer EH, Rapoport TA: Targeting of rough endoplasmic reticulum membrane proteins and ribosomes in invertebrate neurons. Mol Biol Cell 2002;13:1778-1791.

21 Blackstone C: Cellular pathways of hereditary spastic paraplegia. Annu Rev Neurosci 2012;35:25-47.

22 Gray JM, Hill JJ, Bargmann CI: A circuit for navigation in Caenorhabditis elegans. Proc Natl Acad Sci U S A 2005;102:3184-3191.

23 Shorland FB, Jessop AS: Isolation of delta 9 heptadecenoic acid from lamb caul fat. Nature 1955;176:737.

-24 Vlaeminck B, Dufour C, van Vuuren AM, Cabrita AR, Dewhurst RJ, Demeyer D, Fievez V: Use of odd and branched-chain fatty acids in rumen contents and milk as a potential microbial marker. J Dairy Sci 2005;88:1031-1042.

25 Alves SP, Marcelino C, Portugal PV, Bessa RJ: Short communication: The nature of heptadecenoic acid in ruminant fats. J Dairy Sci 2006;89:170-173.

26 Brenner S: The genetics of Caenorhabditis elegans. Genetics 1974;77:71-94.

-27 Zhang Y, Li W, Li L, Li Y, Fu R, Zhu Y, Li J, Zhou Y, Xiong S, Zhang H: Structural damage in the C. elegans epidermis causes release of STA-2 and induction of an innate immune response. Immunity 2015;42:309320.

28 Wang Q, Zhou Y, Song B, Zhong Y, Wu S, Cui R, Cong H, Su Y, Zhang H, He Y: Linking Subcellular Disturbance to Physiological Behavior and Toxicity Induced by Quantum Dots in Caenorhabditis elegans. Small 2016;12:3143-3154.

29 O’Rourke EJ, Soukas AA, Carr CE, Ruvkun G: C. elegans major fats are stored in vesicles distinct from lysosome-related organelles. Cell Metab 2009;10:430-435.

-30 Costa M, Draper BW, Priess JR: The role of actin filaments in patterning the Caenorhabditis elegans cuticle. Dev Biol 1997;184:373-384.

-31 Liu Y, Zhou D, Abumrad NA, Su X: ADP-ribosylation factor 6 modulates adrenergic stimulated lipolysis in adipocytes. Am J Physiol Cell Physiol 2010;298:C921-928.

-32 Wang M, Han RH, Han X: Fatty acidomics: global analysis of lipid species containing a carboxyl group with a charge-remote fragmentation-assisted approach. Anal Chem 2013;85:9312-9320. 